\title{
Doença de Chagas, ambiente, participação e Estado
}

\author{
Chagas disease, environment, \\ participation, and the state
}

João Carlos Pinto Dias 1

1 Centro de Pesquisas René Rachou, Fundação Oswaldo Cruz.

Av. Augusto de Lima 1715, Belo Horizonte, $M G$ 30190-002, Brasil. jcpdias@cpqrr.fiocruz.br

\begin{abstract}
Human Chagas Disease (HCD) affects 16 million Latin Americans and is related to environmental and social questions like education, anthropogenic changes, housing, and migration. HCD requires domiciliation of the vector, which is introduced from sylvatic ecotopes or transported passively by humans. HCD is difficult to treat but possible to prevent. As a disease concentrating among poor individuals and regions, its control requires government intervention, involving education, community participation, and access to medical care. Public policies are required for HCD control, requiring participation by political parties and social control of public agencies to ensure program continuity. A consistent environmental policy is needed to prevent the spread of HCD to new areas such as the Amazon Region. Finally, partnerships among affected countries can help foster HCD control, raising self-esteem and helping promote a new political order in Latin America.
\end{abstract}

Key words Chagas Disease; Insect Vectors; Health Policy; Prevention and Control; Public Health

Resumo Na América Latina, a Doença de Chagas Humana (DCH) afeta 16 milhões de indivíduos e está relacionada a fatores ambientais e sociopolíticos (relações de produção, migrações, vivenda, educação e ação antrópica sobre a natureza). Sua transmissão depende basicamente da domiciliação do vetor originário de ecótopos silvestres ou introduzido na casa por ação humana. De conseqüências graves, a DCH apresenta tratamento difícil, mas boas possibilidades de controle. Como afeta indivíduos e regiões pobres, carece de ação do Estado que envolva educação, participação e políticas públicas de modo a assegurar atenção médica e prevenção. Neste contexto, demanda melhor desempenho político. A política ambiental é fator crítico na prevenção da expansão da DCH para novas áreas de colonização, como a Amazônia. Finalmente, o enfrentamento e controle da DCH na Região pode significar fator de auto-estima e cooperação, entre países, como exercício de novas macropolíticas sociais necessárias ao Terceiro Mundo.

Palavras-chave Doença de Chagas; Política de Saúde; Insetos Vetores; Prevenção e Controle; Saúde Pública 
Na discussão da abordagem sistêmica da saúde humana, a doença de Chagas (DC) representa importante modelo para reflexão no contexto da América Latina, onde significa o 4o maior impacto social entre todas as doenças infeciosas e parasitárias prevalentes (WHO, 1991; Schmunis, 1999). Sua gênese e expansão definem-se, em princípio, por pautas ecológicas e ambientais específicas da entidade primitiva (tripanossomíase americana "silvestre"), na qual circula o agente, Trypanosoma cruzi, há milhares de anos entre insetos vetores (hemípteros reduvídeos) e reservatórios mamíferos em extensos e distintos ambientes naturais americanos. Já as pautas sócio-econômicas e políticas referem-se à doença de Chagas "doméstica”, bem mais recente, que envolve principalmente o homem e vetores domiciliados, em ecótopos artificiais. A tripanossomíase silvestre, por muito antiga, praticamente não produz morbi-mortalidade em dezenas de espécies de mamíferos naturalmente parasitadas, enquanto que a doença de Chagas humana $(\mathrm{DCH})$ apresenta mortalidade importante em sua fase aguda e pode resultar em cardiopatia crônica grave em 10 a 40\% dos indivíduos infectados (Dias \& Coura, 1997).

O panorama atual - com perspectivas concretas de controle da DCH - refere-se, em grande parte, ao balanço ecológico e social entre os ambientes silvestres e domésticos da América Latina, em considerável gama de situações (Forattini, 1980; Dias, 1994). Em sua origem, a DCH dependeu basicamente da domiciliação de vetores infectados provenientes dos ecótopos naturais, ao passo que hoje está dispersa desde o sul dos Estados Unidos até a Patagônia. Neste processo, além da variabilidade da capacidade intrínseca de mais de 120 espécies conhecidas de triatomíneos no que diz respeito a sua adaptação e colonização do ambiente doméstico, entram em jogo prioritariamente fatores humanos e sociais, tais como a qualidade e o tipo de habitação, a ação antrópica sobre o ambiente e as migrações humanas, tudo isto sob forte influência de elementos de natureza política, econômica e cultural, em paralelo com as condições ecológicas e ambientais das diversas microrregiões da área endêmica (Dias, 1994; Schofield, 1994).

De modo geral, a DCH prevalece em espaços ecologicamente "abertos" pelo homem, geralmente respeitando ambientes naturais preservados (Forattini, 1980; Dias, 1994). Assim, a $\mathrm{DCH}$ vai emergir e propagar-se basicamente no seio de populações campesinas pobres da América Latina, não se implantando nos Estados Unidos em virtude da menor capacidade de domiciliação dos vetores locais e do tipo de colonização e de melhores vivendas rurais daquele país (Dias \& Borges Dias, 1979; Dias \& Coura, 1997). A DCH alastrou-se nas áreas rurais da América Latina após a conquista européia, em especial, a partir do século XIX, alcançando seus picos de prevalência e expansão nos meados do século XX. Já o panorama atual mostra uma DCH bastante urbanizada, mercê das migrações rurais-urbanas em todo o Continente, o que aumenta o risco de propagação via transfusional. Também aparecem, nesta evolução, quadros novos, como o da superposição da DCH por AIDS - de conotações epidemiológicas, clínicas e sociais específicas - ou, ainda, a transmissão do T. cruzi por transplantes de órgãos (Dias \& Coura, 1997).

Curiosamente, a doença foi descoberta em 1909 por Carlos Chagas, um brasileiro, e a maior parte de seu conhecimento tem sido desvendada na América Latina, mormente por investigadores do Brasil, da Argentina e da Venezuela. Seu tratamento é complexo e difícil na fase crônica - que afeta 16 milhões de latino-americanos -, não havendo maior interesse comercial para as grandes empresas internacionais no sentido do desenvolvimento de fármacos específicos. O controle da doença, com base em melhoria de habitação, combate químico aos vetores e seleção de doadores de sangue, é viável e depende fundamentalmente de vontade política e de um programa com a continuidade e a contigüidade necessárias. Disto, e por ser a DCH um apanágio de populações pobres ou excluídas, a lógica do controle da endemia passa pela formulação de nova ética social em que o Estado assume e garante a proteção dos cidadãos (saúde como dever do Estado) e não pela lógica das economias de mercado (Dias, 1998, 1999). No Brasil, por pressão social e da comunidade científica, o controle da DCH foi priorizado nos anos 80 e resultou hoje na virtual interrupção da transmissão da doença, a despeito de vários problemas, tais como eventuais faltas de recurso e desvio de pessoal para outros programas, como o do dengue.

$\mathrm{O}$ atual panorama brasileiro é basicamente de vigilância epidemiológica e controle dos bancos de sangue, ao lado do desafio de fornecer atenção médica e previdenciária a cerca de três milhões de infectados. Isto pressupõe, de um lado, o funcionamento do sistema de saúde e, de outro, a participação comunitária, no sentido de que esta vigilância deverá perdurar pelo menos por duas décadas. Naturalmente, a sustentabilidade destas ações dependerá também do amadurecimento político e do crescimento da cidadania entre as populações. Neste 
sentido, entretanto, observa-se que dois setores públicos têm sido ausentes ou omissos no processo, no Brasil e demais países latino-americanos: o sistema de educação e os partidos políticos. O primeiro, em geral, não mobiliza ou liberta a sociedade, pois é mais dependente do que inovador quanto às políticas vigentes, $\mathrm{e}$ não confere cidadania. O ensino público, teoricamente capaz de diminuir iniqüidades, vai sendo atropelado pelo enxugamento do Estado e por interesses que dominam os governos e as elites. O produto da universidade não é capaz ou capacitado a promover reformas políticas ou sociais e não promove uma visão integralizadora do mundo e da sociedade. As pesquisas científicas e tecnológicas são tímidas e insuficientes nos países pobres, em âmbito mundial, e isto só faz crescer as desigualdades. Não obstante, neste ponto, as pesquisas relativas à doença de Chagas fazem exceção em vários lugares da América Latina, resguardando um pouco, de certa forma, a auto-estima e encontrando soluções para um problema concreto da Região que não interessa ao primeiro mundo (Dias, 1998, 1999). Já os partidos políticos latino-americanos, via de regra, fazem basicamente o jogo do poder. São imediatistas por excelência, não formulam ou sustentam programas voltados para o social, o ambiente e a saúde, além de não respaldarem a cidadania em sua prática diuturna (Dias, 1999).

Tecnicamente, os desafios da doença de Chagas no Brasil prendem-se ao cuidado dos já infectados, à qualidade do sistema de saúde (bancos de sangue e transplantes), ao ambiente (espécies vetoras secundárias e nativas, com potencial de domiciliação, em especial, no Nordeste (Caatinga) e na Amazônia (floresta tropical). Dependem, portanto, de macropolíticas que salvaguardem a justiça social (eqüidade, acesso e sustentabilidade das ações em áreas mais pobres) e a preservação do meio ambiente, como pressupostos básicos de saúde pública e à qualidade de vida no planeta. Mais do que inovações tecnológicas, estas grandes questões estão a reclamar cidadania, visão de mundo e ampla participação social, a partir de um Estado minimamente controlador, provedor e redistribuidor (Dias, 1999).

No horizonte operacional, a vigilância epidemiológica sobre a tripanossomíase americana é hoje prioridade no Brasil, devendo ser idealmente horizontalizada, participativa e sustentável por, pelo menos, duas décadas. Trata-se da consolidação, em primeiro lugar, da domiciliação de vetores principais nas áreas de sua dispersão e, a partir disto, da vigilância permanente sobre a possível invasão de veto- res secundários provenientes de ecótopos naturais. Trata ainda da vigilância sobre a qualidade dos bancos de sangue - especificamente o descarte de sangue de doadores infectados, que atualmente representam cerca de $0,7 \%$ dos doadores do Brasil (Schmunis, 1999). No entanto, refere-se ainda à preservação do ambiente natural ou seu manejo racionalizado, como no caso da Amazônia, onde o T. cruzi circula de forma intensa entre reservatórios e vetores silvestres, raramente acometendo o ser humano (Dias, 1994, 1997; Schmunis, 1999). Neste panorama, a DCH comporta-se de modo similar à malária no Brasil, cuja transmissão natural tem sido eficazmente combatida em áreas extra-amazônicas, remanescendo nestas um quadro de vigilância epidemiológica.

Por sua vez, a malária na Amazônia é de difícil controle, com enormes complicadores de ordem ecológica e social, enquanto que a $\mathrm{DCH}$ ainda é toda uma hipótese a depender do manejo ambiental e das políticas sociais de ocupação do espaço e da formulação de estratégias adequadas de saúde pública (Dias, 1998; Dias \& Coura, 1997). Por isto, a questão da DCH na Amazônia não só constitui um problema esperável a demandar atitudes específicas e eminentemente preventivas ao nível das populações e do ambiente, como também serve de modelo e como exercício para uma postura ética e objetiva dos principais protagonistas, inclusive o governo (Dias, 1998).

No plano da DCH extra-amazônica, a evolução de seu controle e de sua epidemiologia, no Brasil, aponta cada vez mais para esquemas descentralizados e para a participação comunitária a curto e médio prazos. Com a urbanização progressiva dos pacientes e a focalização e rareamento dos triatomíneos domiciliados, desaparecem a racionalidade e a eficiência dos programas verticais. Hoje, a total maioria dos focos residuais de triatomíneos é peridomiciliar (galinheiros, cercas, amontoados de telhas e tijolos, pombais etc.) e composta por espécies nativas dos vetores regionais (Triatoma sordida no Sudeste e Centro-Oeste, Triatoma brasiliensis e Triatoma pseudomaculata no Nordeste, Triatoma rubrovaria no Rio Grande do Sul, etc. (Silveira \& Vinhaes, 1998). Estas espécies provêm de ecótopos naturais do cerrado, da caatinga ou da pampa, onde vivem geralmente muito dispersas e em pequenas densidades, sendo sua domiciliação, naturalmente, uma conseqüência da ação antrópica e das condições da vivenda humana, em particular, de seu peridomicílio (Schofield, 1994; Dias, 1997). No peridomicílio implantam-se conforme as condições de alimento e abrigo, formam 
colônias de tamanho variável e, daí, podem invadir as casas e, eventualmente, colonizá-las. Por isto, o peridomicílio funciona, ao mesmo tempo, como barreira e fonte destes triatomíneos nativos na região. Nele, não funcionam bem os inseticidas convencionais (desativação por luz, umidade, poeira e vento), cujo poder residual fica reduzido de modo significativo. Aí requer-se principalmente a participação dos moradores no que toca a sua organização, limpeza e desentulhamento periódicos, com isto diminuindo as oportunidades de colonização triatomínica (Dias, 1997).

Hoje, esquemas simplificados e municipalizados de vigilância foram organizados e implementados por todo o Brasil e em vários outros países, tendo como tônica a notificação de triatomíneos aos sistemas municipais ou regionais de saúde, assim possibilitando o estudo e controle dos focos. A participação comunitária é então fundamental, embasada na informação e na educação, devendo ser atrelada a outros problemas e necessidades da comunidade e dispor de um sistema mínimo de referência e supervisão (Dias, 1998). Mantida e sustentada esta vigilância, a recrudescência da transmissão vetorial da DCH torna-se cada vez menos provável, pois, em baixas densidades ambientais, os triatomíneos apresentam grande dificuldade de colonização domiciliar e aí, por conseqüência, a transmissão da doença é praticamente nula. Inúmeros exemplos disto têm sido registrados em toda a área endêmica, anotando-se, por exemplo, a virtual interrupção da transmissão da DCH em regiões outrora hiperendêmicas, como Bambuí (Minas Gerais) e todo o Estado de São Paulo (Dias, 1997; Dias \& Schofield, 1999). Em decorrência, a eliminação de casos novos por transmissão vetorial em novas gerações irá repercutir nas outras formas de transmissão da DCH - em particular, a transfusional e a congênita - com o esgotamento progressivo de doadores de sangue e gestantes chagásicos, respectivamente (Dias \& Coura, 1997; Schmunis, 1999).

Dos pontos de vista social e econômico, o controle da transmissão da DCH é altamente benéfico, não somente por possibilitar a eliminação de um agravo de grande importância humana, mas também por representar enorme retorno financeiro aos investimentos realizados em termos de economia com atenção médica e previdenciária, de um lado, e da recuperação de dias de trabalho perdidos por morte precoce ou absenteísmo, de outro (Schofield \& Dias, 1991; Schmunis, 1999).

Já no plano político, a efetivação do controle da DCH representa claramente uma "opção social" do Estado e um compromisso do respectivo governo com camadas populacionais marginalizadas. Além disto, no atual contexto de iniciativas intergovernamentais para o controle da DCH - como a do Cone Sul - caminhase para o exercício efetivo de cooperação entre os países envolvidos, com enormes benefícios específicos e desdobramentos na formulação de políticas sociais e de saúde comuns e compartidas, na Região (Dias, 1998; Dias \& Schofield, 1999; Schmunis, 1999). Como perspectiva epidemiológica e política, logrando-se o controle da DCH, desaparecerão os casos agudos da moléstia e ficarão naturalmente cada vez mais escassos os focos de triatomíneos e os doadores e gestantes infectados. Com isto, a importância da DCH tende a decair, ensejando, a planejadores e economistas, a redução dos orçamentos para a necessária vigilância, o que pode redundar em perdas e retrocessos no futuro, sendo este um problema adicional que comprometeria a consolidação de um programa bem sucedido (Dias, 1998; Dias \& Schofield, 1999).

As notas e observações acima mostram a tripanossomíase americana como entidade que emerge da interação biológica de vetores e reservatórios de um microorganismo americano em seus ecótopos naturais diversificados ecologicamente, posteriormente redundando em doença humana de grande impacto social. Observa-se, neste contexto, a evolução do conhecimento a respeito da doença e a formulação de seu controle, propiciando tanto a resolução de um problema médico-social quanto o crescimento científico de várias gerações de pesquisadores latino-americanos, fatos vaticinados e sonhados pelo imortal descobridor da doença, Carlos Ribeiro Justiniano das Chagas (Coutinho \& Dias, 1999; Dias \& Schofield, 1999).

Finalmente, como reflexão final, atente-se que a DCH incide na América Latina, justamente região de transição entre o mundo desenvolvido e muitos povos excluídos e subdesenvolvidos, servindo sua superação como um teste de possibilidades para estes países emergentes em termos de crescimento científico e político social. É um desafio fundamental que se coloca no contexto da DCH, o de vencer as barreiras do subdesenvolvimento e da iniquidade por intermédio do crescimento científico, da participação individual e da coletividade, da construção de nova ética social. Neste sentido, a sustentação de políticas sociais conseqüentes, a participação responsável de todos, a superação de doenças como a DCH e a salvação da natureza irão representar, no novo milênio, indicadores fundamentais dos avanços e fracassos destes povos e de seus governos (Dias, 1999). 


\section{Referências}

COUTINHO, M. \& DIAS, J. C. P., 1999. A reason to celebrate: The saga of Brazilian chagologists. Ciência e Cultura, 51:394-410.

DIAS, J. C. P., 1994. Ecological aspects of the vectorial control of Chagas' disease in Brazil. Cadernos de Saúde Pública, 10 (Sup. 2):352-359.

DIAS, J. C. P., 1997. Controle da doença de Chagas. In: In: Clínica e Terapêutica da Doença de Chagas. Uma Abordagem Prática para o Clínico Geral (J. C. P. Dias \& J. R. Coura, org.), pp. 453-468, Rio de Janeiro: Editora Fiocruz.

DIAS, J. C. P., 1998. Problemas e possibilidades de participação comunitária no controle das grandes endemias no Brasil. Cadernos de Saúde Pública, 14 (Sup. 2): 19-37.

DIAS, J. C. P., 1999. Estado e doenças tropicais. Belo Horizonte: Estado de Minas, 2 dez., Opinião, p. 7.

DIAS, J. C. P. \& BORGES-DIAS, R., 1979. Aspectos sociais, econômicos e culturais da doença de Chagas. Ciência e Cultura, 31 (Sup.):105-118.

DIAS, J. C. P. \& COURA, J. R., 1997. Epidemiologia. In: Clínica e Terapêutica da Doença de Chagas. Uma Abordagem Prática para o Clínico Geral (J. C. P. Dias \& J. R. Coura, org.), pp. 33-66, Rio de Janeiro: Editora Fiocruz.
DIAS, J. C. P. \& SCHOFIELD, C. J., 1999. The evolution of Chagas disease (American Trypanosomiasis) control since Carlos Chagas discovery. Memórias do Instituto Oswaldo Cruz, 94 (Sup. 1):103-151.

FORATTINI, O. P., 1980. Biogeografia, origem e distribuição de triatomíneos no Brasil. Revista de Saúde Pública, 14:265-299.

SCHMUNIS, G. A., 1999. A Tripanossomíase americana e seu impacto na saúde pública das Américas. In: Trypanosoma cruzi e doença de Chagas (Z. Brener, A. A. Andrade \& M. Barral-Netto, org.), pp. 1-15, Rio de Janeiro: Guanabara Koogan Editora.

SCHOFIELD, C. J. \& DIAS, J. C. P., 1991. A cost-benefit analysis of Chagas disease control. Memórias do Instituto Oswaldo Cruz, 86:285-295.

SCHOFIELD, C. J., 1994. Triatominae: Biology and Control. London: Eurocommunica Publications.

SILVEIRA, A. C. \& VINHAES, M. C., 1998. Doença de Chagas: Aspectos epidemiológicos e de controle. Revista da Sociedade Brasileira de Medicina Tropical, 31 (Sup. 2):15-60.

WHO (World Health Organization), 1991. Control of Chagas Disease. Technical Report Series 811. Geneva: WHO. 